\title{
$\mathrm{Mg}$ 掺杂 $\mathrm{TiO}_{2}$ 纳米晶光氧化还原染料的可逆颜色转变研究
}

\author{
赵海兵 ${ }^{1,2}$, 徐海峰 ${ }^{1,2}$, 杨克伟 ${ }^{1,2}$, 林辰学 ${ }^{1,2}$, 冯 苗 ${ }^{1,2}$, 于 岩 ${ }^{1,2}$
}

(福州大学 1. 材料科学与工程学院; 2. 生态环境材料先进技术福建省高等学校重点实验室, 福州 350116)

摘 要: 由于非直接接触、远程控制、高效及快捷的优点, 光可逆颜色转换材料在信息存储、显示器件、传感器等 领域有着重要应用。复合无机材料和有机材料, 实现协同增效, 是新型光驱动可逆颜色转换材料的研究热点之一。 本研究采用一步液相合成法制备粒径约为 $5 \mathrm{~nm}$ 的锐钛矿型 $\mathrm{Mg}^{2+}$ 掺杂 $\mathrm{TiO}_{2}$ 纳米晶, 通过 $\mathrm{X}$ 射线衍射、透射电子显 微镜、 $X$ 射线光电子能谱、红外光谱和拉曼光谱等表征手段确认材料的组成结构, 并对比研究了 $\mathrm{Mg}^{2+}$ 掺杂 $\mathrm{TiO}_{2}$ 对 亚甲基蓝(MB)光可逆颜色转换性能的增强效应。结果表明, 掺杂 $\mathrm{Mg}^{2+}$ 在 $\mathrm{TiO}_{2}$ 晶格中能产生杂质能级, 有效抑制了 光生载流子的复合, 提高了 $\mathrm{TiO}_{2}$ 光氧化还原 $\mathrm{MB}$ 的活性; 另一方面, 掺杂 $\mathrm{Mg}^{2+}$ 降低了 $\mathrm{TiO}_{2}$ 纳米晶的吸收波长, 在 可见光照射下能够有效地抑制 MB 向还原态 $\mathrm{LMB}$ 的转变, 提高系统着色速率。这种基于碱金属掺杂 $\mathrm{TiO}_{2}$ 纳米晶 的光可逆颜色转换材料在许多光电子器件领域具有潜在的应用价值。

关 键 词: $\mathrm{TiO}_{2}$ 纳米晶; 碱金属掺杂; 氧化还原; 亚甲基蓝

中图分类号: TK511 文献标识码: A

\section{Enhanced Photoreversible Color Switching of Methylene Blue Catalyzed by Magnesium-doped $\mathrm{TiO}_{2}$ Nanocrystals}

\author{
ZHAO Hai-Bing $^{1,2}$, XU Hai-Feng ${ }^{1,2}$, YANG Ke-Wei $^{1,2}$, LIN Chen-Xue $^{1,2}$, FENG Miao $^{1,2}$, YU Yan ${ }^{1,2}$ \\ (1. College of Materials Science and Engineering, Fuzhou University, Fuzhou 350116 China; 2. Key Laboratory of Eco-Ma- \\ terials Advanced Technology, Fuzhou University, Fuzhou 350116, China)
}

\begin{abstract}
Because the advantages of non-direct contact, remote control, high efficiency and fastness, light-driven reversible color switching materials (LDRCSMs) have important applications in information storage, display devices, sensors, and other fields. The combination of inorganic materials and organic materials to achieve synergies is one of the research hotspots of new LDRCSMs. A one-step liquid-phase synthesis method was used to prepare Mg-doped $\mathrm{TiO}_{2}$ nanocrystals $\left(\mathrm{Mg}-\mathrm{TiO}_{2} \mathrm{NCs}\right)$ with a particle size of $\sim 5 \mathrm{~nm}$. The reversible light-responsive color switching of methylene blue (MB) by using $\mathrm{Mg}^{-\mathrm{TiO}_{2}} \mathrm{NCs}$ was investigated. The results show that $\mathrm{Mg}^{2+}$ doping generates impurity levels in the $\mathrm{TiO}_{2}$ lattice, effectively inhibiting the recombination of photogenerated carriers and increasing the activity of $\mathrm{TiO}_{2}$ photooxidation-reduced $\mathrm{MB}$. On the other hand, $\mathrm{Mg}^{2+}$ doping can decrease the absorption wavelength of $\mathrm{TiO}_{2}$ $\mathrm{NCs}$, which effectively represses the partial reduction of MB to reduced LMB under visible light irradiation, leading to a higher recoloration rate. Such LDRCSMs based on alkali metal-doped $\mathrm{TiO}_{2} \mathrm{NCs}$ may have potential application in many optoelectronic device fields.
\end{abstract}

Key words: $\mathrm{TiO}_{2}$ nanocrystals; alkali metal doping; redox; methylene blue

收稿日期：2018-02-06; 收到修改稿日期：2018-03-30

基金项目: 国家自然科学基金(51402051，51102047); 福建省高校杰出青年科研人才计划(2016); 福州大学贵重仪器设备开 放测试基金(2018T005)

National Natural Science Foundation of China (51402051, 51102047); Fujian Provincial Outstanding Young Scientists Program (2016); Fuzhou University, Expensive Equipment Open-end Test Fund (2018T005)

作者简介：赵海兵(1991-), 男, 硕士研究生. E-mail: 1913686245@qq.com

通讯作者: 冯 苗, 副教授.E-mail: mfeng@fzu.edu.cn; 于 岩, 教授.E-mail: yuyan@fzu.edu.cn 
光驱动可逆颜色转换材料在光学显示、数据存 储、光电子器件和光传感器等领域表现出广泛的应 用前景, 近年来备受人们的关注 ${ }^{[1-2]}$ 。目前, 光驱动 可逆颜色转换材料主要以具有光致异构效应发色团 的有机染料为主, 如螺恶嗪、苯并吡喃和偶氮苯等, 但由于颜色变换的可逆性和转换速率的限制, 在实 际应用中仍然面临着挑战 ${ }^{[3-5]}$ 。

亚甲基蓝(MB)是一种亮蓝色的阳离子型噻嗪 染料, 具有良好的光化学活性, 在 $660 、 614$ 和 $292 \mathrm{~nm}$ 处有最大吸收。还原态的 MB(LMB) 是无色 的, 最大吸收峰位于 $256 \mathrm{~nm}$, 能稳定存在于脱氧的 水溶液中。将 $\mathrm{MB}$ 氧化还原驱动的颜色转换效应与 $\mathrm{TiO}_{2}$ 纳米晶的光催化性能相结合, 利用非离子聚合 物作为牺牲剂的电子供体(SED)清除光生空穴, 可 开发出新型的光可逆颜色转换材料 ${ }^{[6-7]}$ 。在紫外光照 射下, $\mathrm{TiO}_{2}$ 纳米晶体的光生空穴被表面 SED 分子捕 获, 光生电子将蓝色 $\mathrm{MB}$ 还原为无色 $\mathrm{LMB}$ 。由于还 原态 LMB 很不稳定, 无色 LMB 随后被空气中溶解 的氧重新氧化为蓝色 $M B$, 体系颜色实现由无色恢 复为蓝色的变化过程。尽管 $\mathrm{TiO}_{2}$ 具有优异的化学稳 定性, 但 $\mathrm{TiO}_{2} / \mathrm{MB}$ 溶液的颜色转换效率随着 SED 在 反应过程中的逐渐消耗而降低。掺杂钡离子的 $\mathrm{TiO}_{2}$ 纳米晶可延长该颜色转换体系的使用寿命, 由 $\mathrm{Ba}$ 产生的氧空位有效地清除 UV 照射产生的光空穴, 显著改善了光可逆颜色转换的效率 ${ }^{[8]}$ 。研究发现, 掺 杂碱金属离子可降低 $\mathrm{TiO}_{2}$ 纳米晶体的吸收波长, 在 可见光照射下有效地抑制 MB 向 LMB 的部分还原, 提高系统着色速率。

碱金属 $\mathrm{Mg}$ 与 $\mathrm{Ba}$ 处于同一主族, 且 $\mathrm{Mg}$ 离子半 径远小于 $\mathrm{Ba}$ 离子半径, 相对容易掺杂进入 $\mathrm{TiO}_{2}$ 晶 格中产生氧空位缺陷, 可替代表面牺牲剂的电子供 体, 用于提高 $\mathrm{TiO}_{2}$ 光氧化还原 $\mathrm{MB}$ 的速率。基于以 上分析，本文采用一步液相合成法制备碱金属 $\mathrm{Mg}$ 离子掺杂 $\mathrm{TiO}_{2}$ 纳米晶, 研究 $\mathrm{Mg}$ 离子掺杂对 $\mathrm{TiO}_{2}$ 纳 米晶组成结构和形貌的影响, 探讨 $\mathrm{Mg}$ 掺杂 $\mathrm{TiO}_{2}$ 纳 米晶对 $\mathrm{MB}$ 的光氧化还原颜色转换的增强效应, 为 新型 $\mathrm{TiO}_{2} / \mathrm{MB}$ 光驱动可逆颜色转换材料的研究提供 实验基础和理论依据。

\section{1 实验方法}

\section{1 $\mathrm{Mg}$ 掺杂 $\mathrm{TiO}_{2}$ 纳米晶的制备}

根据文献[8], 当碱金属离子与起始反应物钛源 $\mathrm{TiCl}_{4}$ 的原子比为 $11: 10$ 时, 可制备获得光氧化还原 性能较好的掺杂 $\mathrm{TiO}_{2}$ 纳米晶。据此, 在室温下先将
$2 \mathrm{mmol} \mathrm{MgCl} \mathrm{Mg}_{2} \cdot 6 \mathrm{H}_{2} \mathrm{O}$ 金属盐溶解在 $20 \mathrm{~mL}$ 二甘醇和 $0.2 \mathrm{~mL}$ 去离子水中, 待金属盐完全溶解后将混合溶 液转移至三口烧瓶中并缓慢加热。当温度升至 60 $80^{\circ} \mathrm{C}$ 时, 将 $0.2 \mathrm{~mL} \mathrm{TiCl}_{4}$ 缓慢滴加于反应体系中, 使 $\mathrm{Mg}$ 原子与 $\mathrm{Ti}$ 原子比约为 $11: 10$ 。继续升温至 $220^{\circ} \mathrm{C}$ 后，保持在该反应温度下继续反应 $3 \mathrm{~h}$ 。将反应所得 混合物冷却至室温, 在磁力搅拌条件下加入 $60 \mathrm{~mL}$ 丙酮，并以 $13000 \mathrm{r} / \mathrm{min}$ 的转速离心分离，获得白色 沉淀物。经无水乙醇洗涤 5 次后，为防止纳米晶粒 在干燥过程中团聚，将产物超声分散于 $15 \mathrm{~mL}$ 去离 子水中, 制得均一稳定的 $\mathrm{Mg}-\mathrm{TiO}_{2}$ 胶体悬浮液。按 上述同样步骤，在不加金属盐的条件下，制得纯 $\mathrm{TiO}_{2}$ 胶体悬浮液。

$10 \mathrm{mg} / \mathrm{mL}$ 胶体悬浮液的配制：称量一只塑料 离心管质量(记为 $m_{1}$ ); 向离心管中加入 $3 \mathrm{~mL}$ 胶体悬 浮液后, 将样品冷冻干燥 $12 \mathrm{~h}$ 获得白色粉末, 再次 称量样品与离心管的总质量, 记为 $m_{2}$; 计算 $3 \mathrm{~mL}$ 胶体悬浮液中所含样品的质量 $m=m_{2}-m_{1}$ 。由此可得, $\mathrm{Mg}-\mathrm{TiO}_{2}$ 和纯 $\mathrm{TiO}_{2}$ 胶体悬浮液的浓度分别为 $10.3 \mathrm{mg} / \mathrm{mL}$ 和 $14.2 \mathrm{mg} / \mathrm{mL}$ 。在此基础上, 分别配制 浓度为 $10 \mathrm{mg} / \mathrm{mL}$ 的 $\mathrm{Mg}-\mathrm{TiO}_{2}$ 和纯 $\mathrm{TiO}_{2}$ 胶体悬浮液。

\section{$1.2 \mathrm{Mg}$ 掺杂 $\mathrm{TiO}_{2}$ 纳米晶的表征}

用 Miniflex 600 型 $\mathrm{X}$ 射线衍射仪 $(\mathrm{Cu}$ 靶, $\mathrm{K} \alpha$ 线) 测定 $X$ 射线粉末衍射谱(XRD); 由 $d x r-2 x i$ 型拉曼光 谱仪测得拉曼光谱(Raman); 采用透射电子显微镜 (TEM, TECNAIG2F 20 型)观察样品形貌; 采用 X 射 线衍射光电子能谱仪(XPS, ESALAB 250 型)分析样 品的表面元素组成; 采用 Lambda 950 型分光光度 计测试样品的紫外-可见(UV-Vis)吸收光谱，以硫酸 钡标准白板作为参照; 由 Nicolet 5700 型光谱仪测 得红外光谱(FT-IR); 由 FM-4 型苂光分光光度计测 得光致发光光谱(PL); 由 CHI 660D 电化学工作站 测得光电流曲线 $(i-t)$; 由 SZ-100 型纳米粒子分析仪 测得 Zeta-电位值。

\section{3 光氧化还原性能测试}

将 $2 \mathrm{~mL} \mathrm{Mg}-\mathrm{TiO}_{2}$ 胶体悬浮液 $(\sim 10 \mathrm{mg} / \mathrm{mL})$ 与 $48 \mathrm{~mL} \mathrm{MB}$ 溶液 $\left(2 \times 10^{-5} \mathrm{~mol} / \mathrm{L}\right)$ 混合于 $50 \mathrm{~mL}$ 石英试 管中制备得到 $\mathrm{TiO}_{2} / \mathrm{MB} / \mathrm{H}_{2} \mathrm{O}$ 反应体系。在光化学反 应仪(Xu Jiang, XPA-7)中完成样品紫外光照射反应, 光源为 $300 \mathrm{~W}$ 永灯(配 $365 \mathrm{~nm}$ 的滤波片); 氙灯(北京 畅拓 CHF-XM-500 W 型)为可见光光源(配 $\lambda \geqslant 420 \mathrm{~nm}$ 滤波片)。在光氧化还原反应过程中, 由 SHIMADZU, UV-2450 型光谱仪测试所得 MB 的 UV-Vis 吸收光 谱。所有样品在紫外光照前均预先通氮气除氧, 并 避光搅拌 $15 \mathrm{~min}$, 以达到吸附/脱附平衡。为了对比 
研究, 取相同体积的纯 $\mathrm{TiO}_{2}(\sim 10 \mathrm{mg} / \mathrm{mL})$ 胶体悬浮 液样品, 在同样条件下测试光氧化还原性能。

\section{2 结果与讨论}

\section{1 晶型结构分析}

图 1(a)为纯 $\mathrm{TiO}_{2}$ 和 $\mathrm{Mg}-\mathrm{TiO}_{2}$ 的 XRD 图谱, 位 于 $2 \theta=25.24^{\circ} 、 37.78^{\circ} 、 47.79^{\circ} 、 54.56^{\circ} 、 62.47^{\circ}$ 、 $69.65^{\circ} 、 75.12^{\circ}$ 处的衍射峰与锐铁矿型 $\mathrm{TiO}_{2}$ (JCPDS 21-1272) 的衍射峰吻合, 说明样品均为锐钛矿型 $\mathrm{TiO}_{2}{ }^{[9-10]}$ 。由此可见, $\mathrm{Mg}^{2+}$ 的掺杂并未改变 $\mathrm{TiO}_{2}$ 的晶 型结构, 但是 $\mathrm{Mg}-\mathrm{TiO}_{2}$ 的衍射峰强度降低, 衍射峰 变宽, 说明 $\mathrm{Mg}^{2+}$ 的掺杂抑制了 $\mathrm{TiO}_{2}$ 晶粒生长 ${ }^{[11]}$ 。

XRD 分析结果列于表 1 。根据谢乐公式: $D=$ $0.89 \lambda / \beta \cos \theta$ ( $D$ 为晶粒尺寸, $\lambda$ 为入射线波长, $\beta$ 为衍 射峰的半高宽)可知, $\mathrm{Mg}-\mathrm{TiO}_{2}$ 与 $\mathrm{TiO}_{2}$ 纳米晶的晶粒
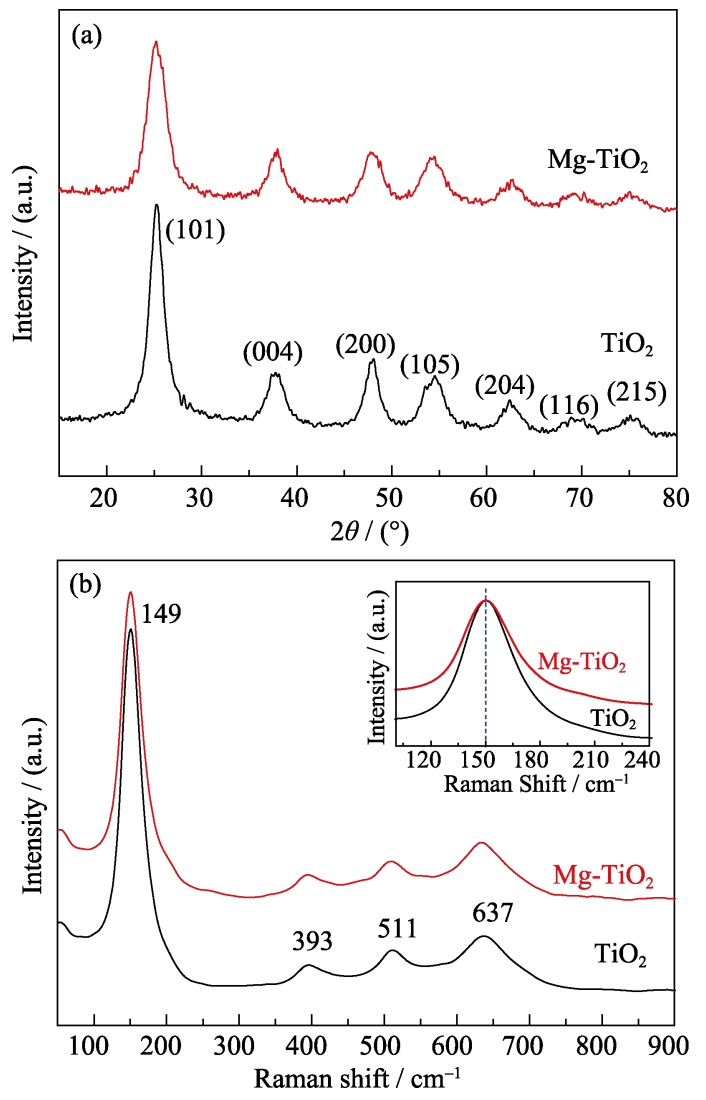

图 1 纯 $\mathrm{TiO}_{2}$ 和 $\mathrm{Mg}-\mathrm{TiO}_{2}$ 的 $\mathrm{XRD}(\mathrm{a})$ 和 $\operatorname{Raman}(\mathrm{b})$ 图谱

Fig. 1 XRD patterns (a) and Raman spectra (b) of pure $\mathrm{TiO}_{2}$ and $\mathrm{Mg}-\mathrm{TiO}_{2} \mathrm{NC}$

表 1 纯 $\mathrm{TiO}_{2}$ 和 $\mathrm{Mg}-\mathrm{TiO}_{2}$ 的 XRD 分析结果 Table 1 XRD analysis results of pure $\mathrm{TiO}_{2}$ and $\mathrm{Mg}-\mathrm{TiO}_{2}$

\begin{tabular}{ccccc}
\hline Sample & $\begin{array}{c}\text { Particle } \\
\text { size } D / \mathrm{nm}\end{array}$ & $\begin{array}{c}\text { Interplanar } \\
\text { spacing, } d / \mathrm{nm}\end{array}$ & $\begin{array}{c}\text { Cell parameters } \\
a=b / \mathrm{nm}\end{array}$ & $2 \theta /\left(^{\circ}\right)$ \\
\hline $\mathrm{TiO}_{2}$ & 5.21 & 0.35238 & 0.038052 & 25.253 \\
$\mathrm{Mg}^{-\mathrm{TiO}_{2}}$ & 4.54 & 0.35173 & 0.037950 & 25.273 \\
\hline
\end{tabular}

尺寸分别为 $4.54 \mathrm{~nm}$ 和 $5.21 \mathrm{~nm}$, 前者晶粒尺寸明显 小于后者, 这是由于 $\mathrm{Mg}^{2+}$ 的半径 $(0.057 \mathrm{~nm})$ 小于 $\mathrm{Ti}^{4+}$ 半径 $(0.061 \mathrm{~nm}), \mathrm{Mg}^{2+}$ 取代 $\mathrm{Ti}^{4+}$ 后引起晶格收缩, 导致晶粒尺寸减小 ${ }^{[12]}$ 。

从样品的 Raman 光谱图(图 1(b)) 可以看出, $\mathrm{Mg}-\mathrm{TiO}_{2}$ 和纯 $\mathrm{TiO}_{2}$ 纳米晶的拉曼图谱基本一致, 四 个峰分别位于 $149 、 393 、 511$ 和 $637 \mathrm{~cm}^{-1}$, 归属于 锐钛矿型 $\mathrm{TiO}_{2}$ 的 Raman 振动峰 ${ }^{[13]}$ 。其中, $149 \mathrm{~cm}^{-1}$ 处的吸收峰对应对称性 Ti-O-Ti 键吸收峰, 该峰在 $\mathrm{Mg}-\mathrm{TiO}_{2}$ 样品中没有明显移动, 这可能与 $\mathrm{Mg}^{2+}$ 掺杂量 较少或掺杂离子高度分散于 $\mathrm{TiO}_{2}$ 晶格中有关 ${ }^{[14-15]}$ 。 但与未掺杂 $\mathrm{TiO}_{2}$ 样品相比, $\mathrm{Mg}-\mathrm{TiO}_{2}$ 峰型明显宽化 (图 1(b)), 说明 $\mathrm{Mg}^{2+}$ 的掺杂加剧了 $\mathrm{TiO}_{2}$ 晶体内部的 无序性 ${ }^{[16]}$ 。

图 2 为 $\mathrm{Mg}-\mathrm{TiO}_{2}$ 和 $\mathrm{TiO}_{2}$ 在不同放大倍数下的 TEM 照片, 可以看出, 二者均为无规则的细小颗粒 状，但 $\mathrm{Mg}-\mathrm{TiO}_{2}$ 比 $\mathrm{TiO}_{2}$ 分散得更均匀，并未出现明 显的颗粒团聚现象。随机取 50 个晶粒尺寸计算样品 的平均晶粒尺寸约 $4.63 \mathrm{~nm}$, 与 XRD 计算结果基本 一致。 $\mathrm{Mg}-\mathrm{TiO}_{2}$ 的条纹间距 $d=0.350 \mathrm{~nm}$, 对应锐钛 矿型 $\mathrm{TiO}_{2}(101)$ 晶面的晶面间距 ${ }^{[17]}$, 这与 XRD 图谱 (图 1(a))中(101)的衍射峰最强结果相对应, 并且条 纹间距略小于纯 $\mathrm{TiO}_{2}(d=0.354 \mathrm{~nm})$, 可能与 $\mathrm{Mg}^{2+}$ 掺杂进入 $\mathrm{TiO}_{2}$ 晶格中破坏了 $\mathrm{TiO}_{2}$ 的微观结构有关 ${ }^{[12]}$ 。 图 2(a)插图为 $\mathrm{Mg}^{-\mathrm{TiO}_{2}}$ 的选区电子衍射图, 所呈现出 的衍射环对应着锐钛矿型 $\mathrm{TiO}_{2}$ 的特征衍射环，这表 明所合成样品为结晶性良好的锐钛矿型多晶 $\mathrm{TiO}_{2}$ 。

\section{2 化学组成分析}

图 3(a)为纯 $\mathrm{TiO}_{2}$ 和 $\mathrm{Mg}-\mathrm{TiO}_{2}$ 的 Ti2p XPS 图谱。

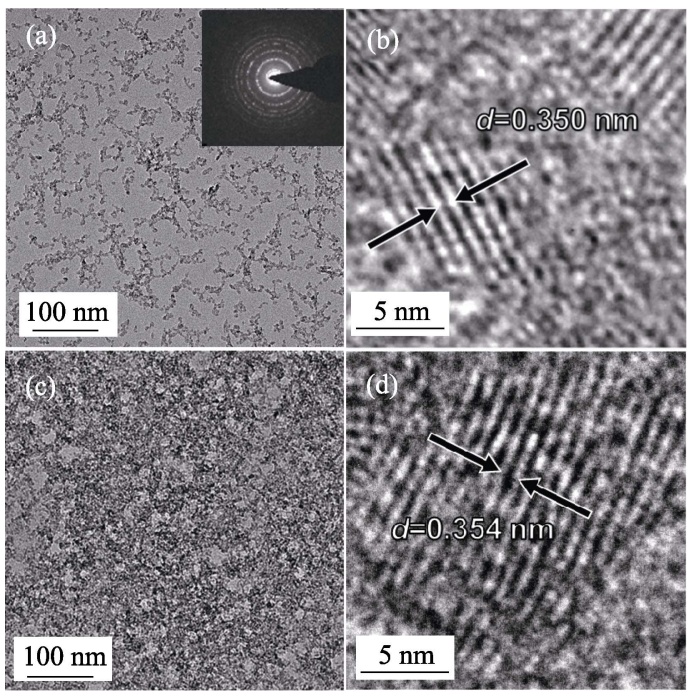

图 $2 \mathrm{Mg}-\mathrm{TiO}_{2}(\mathrm{a}) \sim(\mathrm{b})$ 和 $\mathrm{TiO}_{2}$ (c) (d)不同放大倍数的 TEM 照片 Fig. 2 TEM images at different magnifications of $(a, b)$ $\mathrm{Mg}-\mathrm{TiO}_{2}$ and (c, d) $\mathrm{TiO}_{2}$ 

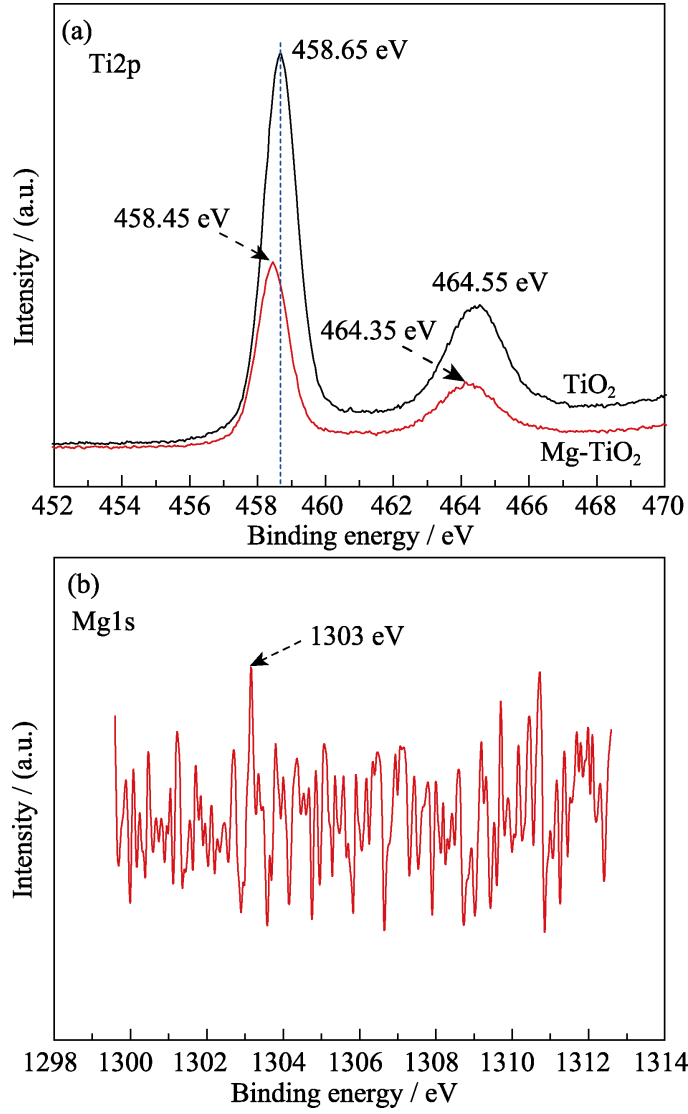

图 3 纯 $\mathrm{TiO}_{2}$ 和 $\mathrm{Mg}-\mathrm{TiO}_{2}$ 的 $\mathrm{Ti} 2 \mathrm{p}$ XPS 图谱(a); $\mathrm{Mg}-\mathrm{TiO}_{2}$ 样 的 Mg1s XPS 图谱(b)

Fig. 3 Ti2p XPS spectra (a) of pure $\mathrm{TiO}_{2}$ and $\mathrm{Mg}-\mathrm{TiO}_{2}$, and (b) $\mathrm{Mg} 1 \mathrm{~s}$ XPS spectrum (b) of $\mathrm{Mg}-\mathrm{TiO}_{2}$

纯 $\mathrm{TiO}_{2}$ 在 458.65 和 $464.55 \mathrm{eV}$ 处的两个吸收峰归属 于 $\mathrm{Ti} 2 \mathrm{p}_{3 / 2}$ 和 $\mathrm{Ti} 2 \mathrm{p}_{1 / 2}$, 表明 $\mathrm{Ti}$ 元素主要以 $\mathrm{Ti}^{4+}$ 形式 存在于 $\mathrm{TiO}_{2}$ 晶格中。 $\mathrm{Mg}-\mathrm{TiO}_{2}$ 的 Ti2p XPS 吸收峰 向低结合能偏移了 $0.2 \mathrm{eV}$, 这可能是由于 $\mathrm{Mg}$ 的电负 性小于 $\mathrm{Ti}$, 当 $\mathrm{Mg}$ 掺杂进入 $\mathrm{TiO}_{2}$ 晶格中形成 $\mathrm{Mg}-\mathrm{O}-\mathrm{Ti}$ 键, 诱导 $\mathrm{O}-\mathrm{Ti}$ 键上的电子向 $\mathrm{Ti}$ 偏移, 从而引起 $\mathrm{Ti} 2 \mathrm{p}$ 的吸收峰向低结合能偏移 ${ }^{[18]}$ 。从 $\mathrm{Mg} 1 \mathrm{~s}$ 的 XPS 图谱
(图 3(b)) 可以看出, $1303 \mathrm{eV}$ 处出现了 $\mathrm{Mg} 1 \mathrm{~s}$ 峰, 但是 强度较弱, 这是因为 XPS 测试是通过 X 射线去辐照 固体样品表面, 激发出光电子从而得到元素定性定 量和化学态信息。Mg1s 结合能较高 $(\sim 1303 \mathrm{eV}), \mathrm{X}$ 光激发的 $\mathrm{Mg} 1 \mathrm{~s}$ 光电子动能较低 ${ }^{[19]}$, 因此 XPS 对 $\mathrm{Mg} 1 \mathrm{~s}$ 的原子灵敏度因子较低, 仅为 3.5。对于原子 序数大的碱金属元素, 例如 $\mathrm{Ba}$ 元素, Ba3d 其结合能 较低 $(\sim 781 \mathrm{eV})$, XPS 对 Ba3d 的原子灵敏度因子为 7.9 , 是 $\mathrm{Mg} 1 \mathrm{~s}$ 的 2.26 倍。因此与 $\mathrm{Ba}$ 掺杂 $\mathrm{TiO}_{2}$ 文献相

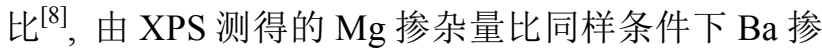
杂量(1.2\%)低。由 XPS 数据分析可得, $\mathrm{Mg}$ 相对于 $\mathrm{Ti}$ 的原子百分比约为 $0.21 \%$ 。据报道, 在 $\mathrm{TiO}_{2-x}$ 薄膜 中即使掺杂量仅为 $0.1 \%$ 也可获得 $5 \times 10^{19} / \mathrm{cm}^{3}$ 电子 供体 ${ }^{[8,20]}$ 。在 $\mathrm{Mg}$ 掺杂的 $\mathrm{TiO}_{2}$ 纳米晶中, 氧空位作 为有效的 SED 可以促进基于氧化还原的颜色转换 材料所需的电荷分离。

\section{3 吸收光谱分析}

样品的 FT-IR 测试结果如图 4(a)所示, Ti-O-Ti 键振动峰位于 $\sim 575 \mathrm{~cm}^{-1}$ 。相比于纯 $\mathrm{TiO}_{2}$ 纳米晶, $\mathrm{Mg}-\mathrm{TiO}_{2}$ 在该处的吸收峰强度有所降低, 且有一定 的红移, 说明 $\mathrm{Mg}^{2+}$ 的掺杂破坏了 $\mathrm{TiO}_{2}$ 晶格的有序 程度。这与 Raman 分析结果相一致 ${ }^{[21]}$ 。在 $\sim 500 \mathrm{~cm}^{-1}$ 处出现了 $\mathrm{Mg}-\mathrm{O}-\mathrm{Ti}$ 键的较弱吸收峰, 进一步证明 $\mathrm{Mg}^{2+}$ 掺杂进入 $\mathrm{TiO}_{2}$ 晶格中 ${ }^{[13]}$ 。

由纯 $\mathrm{TiO}_{2}$ 和 $\mathrm{Mg}-\mathrm{TiO}_{2}$ 纳米晶的 UV-Vis 吸收光 谱图(图 4(b))可知，二者在 200 380 nm 范围内的紫 外光区都有较强的吸收, 归属于 $\mathrm{TiO}_{2}$ 的本征带隙跃 迁(电子由价带 $\mathrm{O} 2 \mathrm{p}$ 轨道跃迁至导带 Ti2p 轨道), 且 $\mathrm{Mg}^{2+}$ 的掺杂引起 $\mathrm{TiO}_{2}$ 吸收带边蓝移 $(15 \mathrm{~nm})$ 。根据 Kubelka-Munk 函数 $(\alpha h v)^{1 / 2}=\mathrm{A}\left(h v-E_{\mathrm{g}}\right)^{1 / 2}$ 与光子能量 的关系图(图 4(b)插图), 计算 $\mathrm{TiO}_{2}$ 纳米晶的能带值 ${ }^{[22]}$ 。 纯 $\mathrm{TiO}_{2}$ 的带隙值为 $3.20 \mathrm{eV}$, 与锐钛矿型 $\mathrm{TiO}_{2}$ 的能
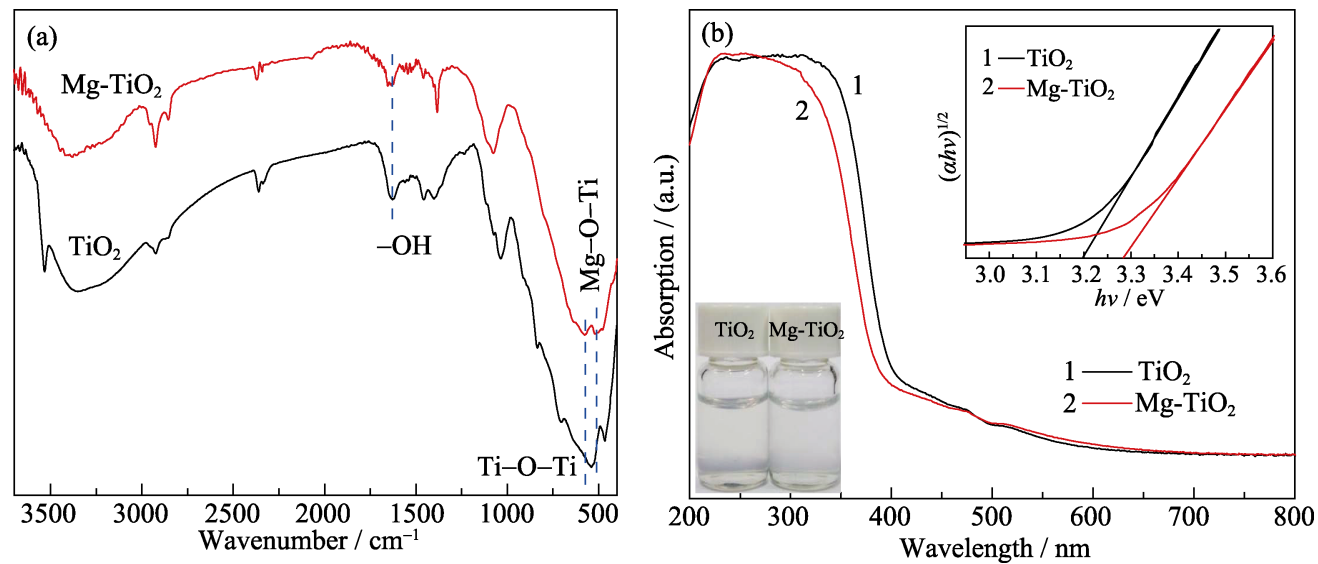

图 $4 \mathrm{TiO}_{2}$ 和 $\mathrm{Mg}-\mathrm{TiO}_{2}$ 的 FT-IR 谱图(a)和 UV-Vis 谱图(样品浓度均为 $10 \mathrm{mg} / \mathrm{mL}$ )(b)

Fig. 4 FT-IR spectra (a) and UV-Vis absorption spectra (b) of pure $\mathrm{TiO}_{2}$ and $\mathrm{Mg}-\mathrm{TiO}_{2}$ Inset: Mott-Schottky plots and pictures of pure $\mathrm{TiO}_{2}$ and $\mathrm{Mg}-\mathrm{TiO}_{2}$ 
带值相一致。 $\mathrm{Mg}-\mathrm{TiO}_{2}$ 纳米晶的能带值为 $3.27 \mathrm{eV}$, 相比于未掺杂的有一定增加, 这是由于 $\mathrm{Mg}$ 掺杂 $\mathrm{TiO}_{2}$ 的晶粒尺寸相对较小, 引起量子尺寸效应所致 ${ }^{[23]}$ 。

图 4(b)插图是两个浓度均为 $10 \mathrm{mg} / \mathrm{mL}$ 的样品悬浮 液，分散性良好，无色透明。

\section{4 光氧化还原性能分析}

图 5(a)和(c)分别为纯 $\mathrm{TiO}_{2}$ 和 $\mathrm{Mg}-\mathrm{TiO}_{2}$ 纳米晶与 $M B$ 混合溶液在紫外光照射下 UV-Vis 吸收光谱图。 在紫外光照射下, $\mathrm{TiO}_{2}$ 被激发产生光生电子空穴对。 光生空穴被表面氧空位缺陷捕获, 剩余的光生电子 从价带跃迁到导带转移给吸附在 $\mathrm{TiO}_{2}$ 表面的 $\mathrm{MB}$ 分 子, 使蓝色 MB 还原为无色 $\mathrm{LMB}$; 在可见光照射下, 将空气通入反应液 (鼓泡速率约为 $2 \sim 3 / \mathrm{s}$ ), 无色 $\mathrm{LMB}$ 与 $\mathrm{O}_{2}$ 接触, 在 $\mathrm{TiO}_{2}$ 光催化诱导作用下将电子 转移至 $\mathrm{O}_{2}$ 生成 $\mathrm{O}_{2}{ }^{-}$发生氧化反应, $\mathrm{LMB}$ 被氧化至 $\mathrm{MB}$ 状态, 如此完成一次光催化氧化还原 $\mathrm{MB}$ 的颜 色可逆变换过程。 $\mathrm{Mg}-\mathrm{TiO}_{2}$ 光还原蓝色 $\mathrm{MB}$ 至无色 LMB 只需 $20 \mathrm{~s}$, 随后在可见光照射下, 光催化氧化 无色 $\mathrm{LMB}$ 至蓝色 MB 用时 $18 \mathrm{~min}$ 。而在相同的条件 下, 纯 $\mathrm{TiO}_{2}$ 完成这两个过程分别需要 $25 \mathrm{~s}$ 和 $24 \mathrm{~min}$,
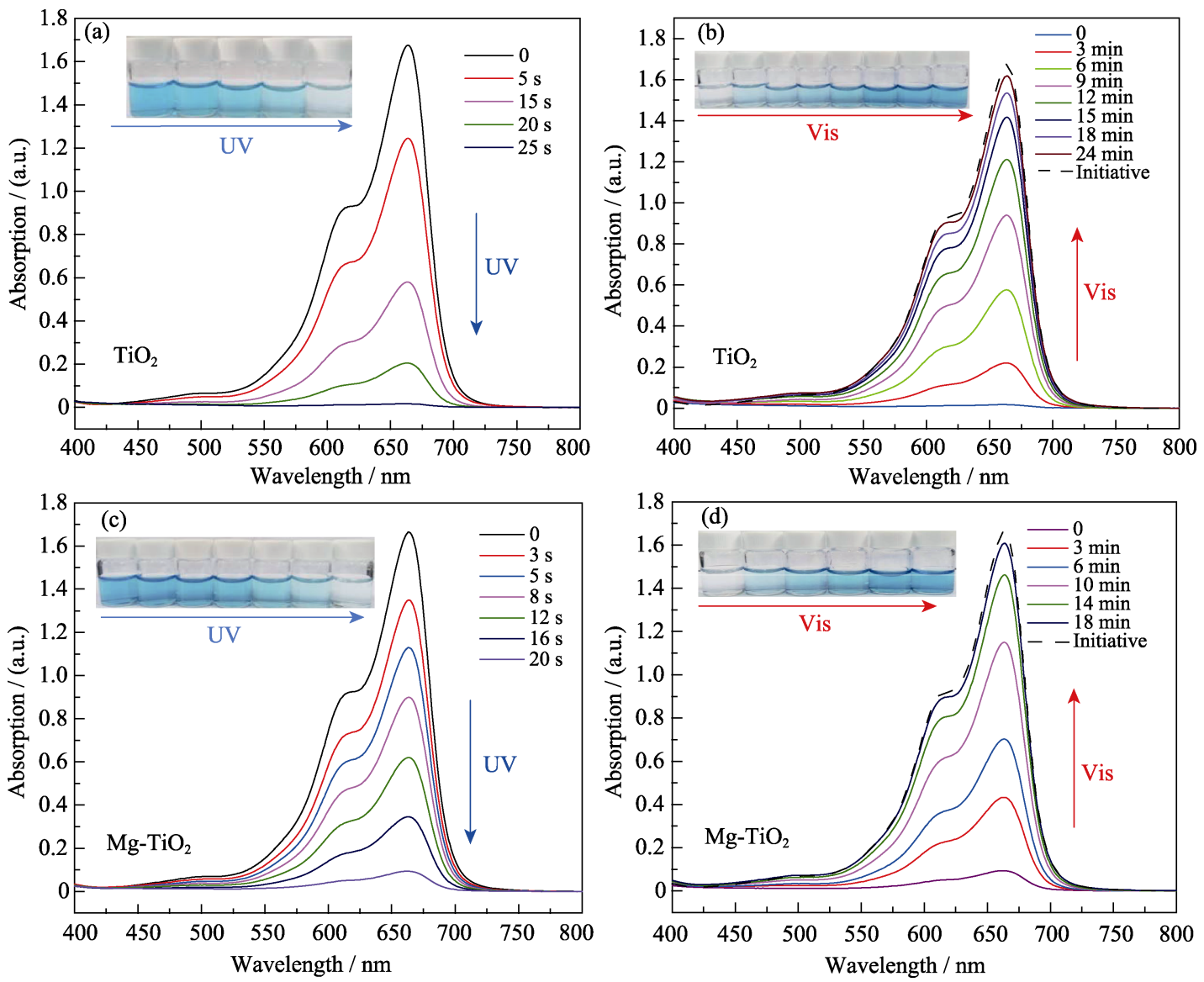

图 5 紫外光照射下 $M B$ 褪色过程的 UV-Vis 吸收光谱图((a) $\left.\mathrm{TiO}_{2},(\mathrm{c}) \mathrm{Mg}-\mathrm{TiO}_{2}\right)$; 可见光照射下着色过程的 UV-Vis 吸收光谱图((b) $\left.\mathrm{TiO}_{2},(\mathrm{~d}) \mathrm{Mg}-\mathrm{TiO}_{2}\right)$

Fig. 5 UV-Vis spectra showing the decoloration process of MB under UV irradiation ((a) $\mathrm{TiO}_{2}$, (c) $\mathrm{Mg}-\mathrm{TiO}_{2}$ ); UV-Vis spectra showing the recoloration process of $\mathrm{MB}$ under visible light irradiation ((b) $\mathrm{TiO}_{2},(\mathrm{~d}) \mathrm{Mg}_{-} \mathrm{TiO}_{2}$ ) Inset: the pictures of the reaction system solutions changing with the illumination time 

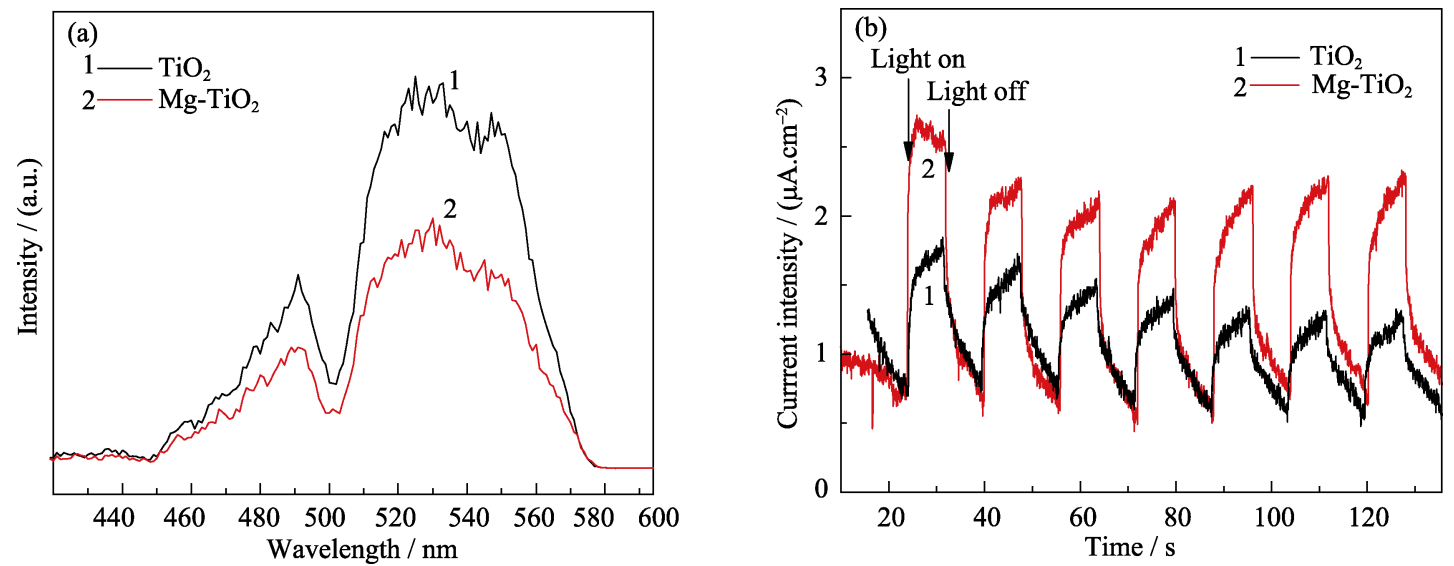

图 6 纯 $\mathrm{TiO}_{2}$ 和 $\mathrm{Mg}-\mathrm{TiO}_{2}$ 纳米晶的 PL 图(a)和 $i-t$ 图(b)

Fig. 6 Fluorescence spectra (a) and photocurrent intensity (b) of pure $\mathrm{TiO}_{2}$ and $\mathrm{Mg}-\mathrm{TiO}_{2}$

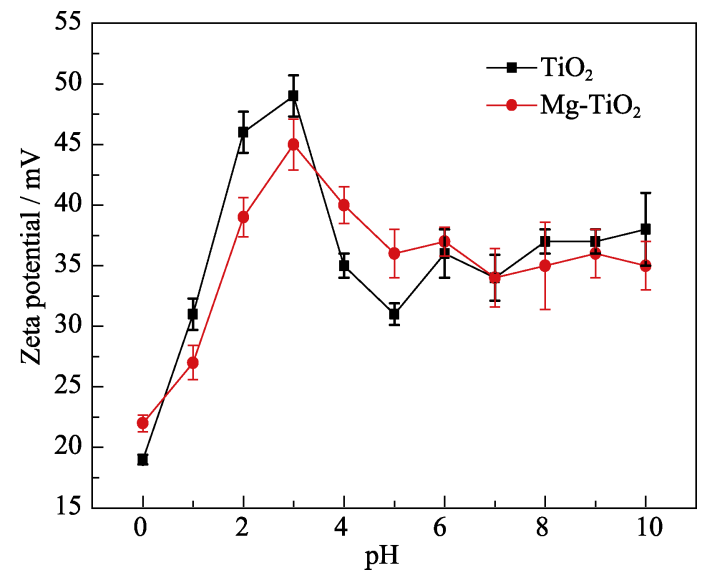

图 7 纯 $\mathrm{TiO}_{2}$ 和 $\mathrm{Mg}-\mathrm{TiO}_{2} \mathrm{NCs}$ 的 Zeta 电位测试结果

Fig. 7 Zeta-potential of pure $\mathrm{TiO}_{2}$ and $\mathrm{Mg}-\mathrm{TiO}_{2} \mathrm{NCs}$

在不同 $\mathrm{pH}$ 条件下的Zeta-电位测试结果表明(图 7), $\mathrm{TiO}_{2}$ 和 $\mathrm{Mg}-\mathrm{TiO}_{2}$ 表面都带正电, 介于 19 49 $\mathrm{mV}$ 之 间, 且 $\mathrm{pH}$ 对二者 Zeta-电位的影响趋势基本一致。 当 $\mathrm{pH}<3$ 时, 样品的表面带电量随 $\mathrm{pH}$ 的增加而增加; 当 $\mathrm{pH}=3$ 时, 表面带电量达到最大值, 分别为 $49 \mathrm{mV}$ $\left(\mathrm{TiO}_{2}\right)$ 和 $45 \mathrm{mV}\left(\mathrm{Mg}-\mathrm{TiO}_{2}\right)$; 当 $3<\mathrm{pH}<5$ 时, 表面带电量 随 $\mathrm{pH}$ 的增加而减小; 当 $5<\mathrm{pH}<10$ 时, 表面带电量趋 于稳定, 不随 $\mathrm{pH}$ 的增加而发生明显变化。颗粒之间 的范德华力和粒子表面静电排斥力的平衡作用 ${ }^{[27]}$, 使 $\mathrm{Mg}-\mathrm{TiO}_{2}$ 纳米晶能够稳定的悬浮于水中(图 4(b)插图)。 总体而言, $\mathrm{Mg}^{-} \mathrm{TiO}_{2}$ 纳米晶的表面带电量受 $\mathrm{pH}$ 的影 响小于未掺杂 $\mathrm{TiO}_{2}$ 纳米晶, 表现出相对稳定的表面 带电性, 有利于提高样品对染料分子的光氧化还原 效应的循环稳定性。

\section{3 结论}

本文报道了一种基于 $\mathrm{Mg}^{2+}$ 掺杂 $\mathrm{TiO}_{2}$ 纳米晶(粒 径约为 $5 \mathrm{~nm}$ )的光催化活性和亚甲基蓝(MB)的氧化
还原驱动颜色转变的新型光驱动可逆颜色转换材 料。该材料在紫外光下从蓝色迅速变为无色, 并在可 见光照射下恢复其原始蓝色。掺杂 $\mathrm{Mg}^{2+}$ 在 $\mathrm{TiO}_{2}$ 晶格 中产生了杂质能级，能有效抑制光生载流子的复合, 提高 $\mathrm{TiO}_{2}$ 光氧化还原 $\mathrm{MB}$ 的活性, 使其可逆颜色转变 效应增强; 另一方面，掺杂 $\mathrm{Mg}^{2+}$ 可以降低 $\mathrm{TiO}_{2}$ 纳米晶 的吸收波长，在可见光照射下能够有效地抑制 $\mathrm{MB}$ 向还原态 LMB 的转变, 提高可逆颜色变换速率。这 种基于碱金属掺杂 $\mathrm{TiO}_{2}$ 纳米晶的光驱动可逆颜色转 换材料在许多光电子器件领域具有潜在的应用价值。

\section{参考文献:}

[1] HAN D, JIANG B L, FENG J, et al. Photocatalytic self-doped $\mathrm{SnO}_{2-x}$ nanocrystals drive visible-light-responsive color switching. Angew. Chem. Int. Ed., 2017, 56(27): 7792-7796.

[2] WANG W S, FENG J, YE Y F, et al. Photocatalytic color switching of transition metal hexacyanometalate nanoparticles for highperformance light-printable rewritable paper. Nano Lett., 2017, 17(2): 755-761.

[3] ZHANG J, ZOU Q, TIAN H. Photochromic materials: more than meets the eye. Adv. Mater., 2013, 25(3): 378-399.

[4] DONG H, ZHU H, MENG Q, et al. Organic photoresponse materials and devices. Chem. Soc. Rev., 2012, 41(5): 1754-1808.

[5] WANG M, SUN L, LIN Z Q, et al. p-n Heterojunction photoelectrodes composed of $\mathrm{Cu}_{2} \mathrm{O}$-loaded $\mathrm{TiO}_{2}$ nanotube arrays with enhanced photoelectrochemical and photoelectrocatalytic activities. Energy \& Environ. Sci., 2013, 6(4): 1211-1220.

[6] WANG W S, YY M M, HE L, et al. Nanocrystalline $\mathrm{TiO}_{2}$-catalyzed photoreversible color switching. Nano Lett., 2014, 14(3): 1681-1686.

[7] WANG W S, XIE N, HE L, et al. Photocatalytic colour switching of redox dyes for ink-free light-printable rewritable paper. Nat. Commun., 2014, 5: 5459.

[8] WANG W S, YE Y F, FENG J, et al. Enhanced photoreversible color switching of redox dyes catalyzed by barium-doped $\mathrm{TiO}_{2}$ nanocrystals. Angew. Chem. Int. Ed., 2015, 54(4): 1321-1326.

[9] LIU X P, CHEN Y Y, CAO C L, et al. Electrospun nitrogen and carbon co-doped porous $\mathrm{TiO}_{2}$ nanofibers with high visible light photocatalytic activity. New J. Chem., 2015, 39(9): 6944-6950. 
[10] RAN H L, HUANG H, MA M J, et al. Dye-sensitized solar cells based on double-layer composite film with enhanced photovoltaic performance. Journal of Inorganic Materials, 2017, 32(10): 1049-1054.

[11] ROY N, SOHN Y, LEUNG K T, et al. Engineered electronic states of transition metal doped $\mathrm{TiO}_{2}$ nanocrystals for low overpotential oxygen evolution reaction. J. Phys. Chem. C, 2014, 118(51): 29499-29506.

[12] WU M C, CHIH J S, HUANG W K. Bismuth doping effect on $\mathrm{TiO}_{2}$ nanofibres for morphological change and photocatalytic performance. CrystEngComm, 2014, 16(46): 10692-10699.

[13] LONG L, ZHANG A, YANG J, et al. A green approach for preparing doped $\mathrm{TiO}_{2}$ single crystals. ACS Appl. Mater. Interfaces, 2014, 6(19): 16712-16720.

[14] CHEN S F, WEI Z, WEI L, et al. Preparation, characterization and activity evaluation of $\mathrm{p}-\mathrm{n}$ junction photocatalyst $\mathrm{p}-\mathrm{CaFe}_{2} \mathrm{O}_{4} / \mathrm{n}-\mathrm{ZnO}$. Chem. Eng. J., 2009, 155(1/2): 466-473.

[15] MIAO L, TANEMURA S, HUANG R, et al. Large Seebeck coefficients of protonated titanate nanotubes for high-temperature thermoelectric conversion. ACS Appl. Mater. Interfaces, 2010, 2(8): 2355-2359.

[16] NALDONI A, ALLIETA M, SANTANGELO S, et al. Effect of nature and location of defects on bandgap narrowing in black $\mathrm{TiO}_{2}$ nanoparticles. J. Am. Chem. Soc., 2012, 134(18): 7600-7603.

[17] KIRAN V, SAMPATH S. Facile synthesis of carbon doped $\mathrm{TiO}_{2}$ nanowires without an external carbon source and their opto-electronic properties. Nanoscale, 2013, 5(21): 10646-10652.

[18] JIN C, ZHENG R Y, GUO Y, et al. Hydrothermal synthesis and characterization of phosphorous-doped $\mathrm{TiO}_{2}$ with high photocatalytic activity for methylene blue degradation. J. Mol. Catal. A: Chem., 2009, 313(1/2): 44-48.

[19] ZHANG X Y, ZHAO Z J, LIU F. Effects of sample height on XPS.
Analysis and Testing Technology and Instruments, 2013, 19(4): 244-246.

[20] HU C C, LIN M S, WU T Y, et al. Near Room-temperature Liquidphase Deposition of Barium-doped $\mathrm{TiO}_{2}$ on n-GaN and Its Application to AlGaN/GaN MOSHEMTs. Physics and Technology of High-K Materials 9, Vol. 41 (Eds.: KAR S, VANELSHOCHT S, KITA K, et al.) The Electrochemical Society, Pennington, New Jersey, USA, 2011: 439-444.

[21] SOOD S, UMAR A, MEHTA S K, et al. Efficient photocatalytic degradation of brilliant green using $\mathrm{Sr}$-doped $\mathrm{TiO}_{2}$ nanoparticles. Ceram. Int., 2015, 41(3): 3533-3540.

[22] REYES-CORONADO D, RODRIGUEZ-GATTORNO G, ESPINOSAPESQUEIRA M E, et al. Phase-pure $\mathrm{TiO}_{2}$ nanoparticles: anatase, brookite and rutile. Nanotechnology, 2008, 19(14): 145605-1-10.

[23] VENKATACHALAM N, PALANICHAMY M, ARABINDOO B, et al. Alkaline earth metal doped nanoporous $\mathrm{TiO}_{2}$ for enhanced photocatalytic mineralisation of bisphenol-A. Catal. Commun., 2007, 8(7): 1088-1093.

[24] XIAO Q, OUYANG L, GAO L, et al. One-step hydrothermal preparation and photocatalytic activity of (C, S, Sm)-tridoped mesoporous $\mathrm{TiO}_{2}$ photocatalyst under visible light irradiation. $M a$ ter. Chem. Phys., 2010, 124(2/3): 1210-1215.

[25] ZHANG L, REISNER E, BAUMBERG J J. Al-doped ZnO inverse opal networks as efficient electron collectors in $\mathrm{BiVO}_{4}$ photoanodes for solar water oxidation. Energy Environ. Sci., 2014, 7(4): 1402-1408.

[26] BLEDOWSKI M, WANG L, RAMAKRISHNAN A. et al. Visiblelight photocurrent response of $\mathrm{TiO}_{2}$-polyheptazine hybrids: evidence for interfacial charge-transfer absorption. Phys. Chem. Chem. Phys., 2011, 13: 21511-21519.

[27] SAHU M, SUTTIPONPARNIT K, SUVACHITTANONT S, et al. Characterization of doped $\mathrm{TiO}_{2}$ nanoparticle dispersions. Chem. Eng. Sci., 2011, 66(15): 3482-3490. 